\title{
Application of Radiation Protection of Patients in Medical Diagnostic Radiology (Case of Public Hospitals, Fianarantsoa District, Haute Matsiatra Region, Madagascar)
}

\author{
Said Soudjay 1, 2, *, Vololoniaina Bernardine ${ }^{2}$, Ramanandraibe Marie Jeanne ${ }^{2,4}$, \\ Ramanantsizehena Georgette Vololona ${ }^{3}$, Raboanary Roland ${ }^{3}$, Randriamora Tiana Harimalala ${ }^{2}$ \\ ${ }^{1}$ Department of Physics, Faculty of Sciences and Technologies, University of Comoros, Moroni, Comoros \\ ${ }^{2}$ Department of Dosimetry and Radiation Protection, National Institute of Sciences and Nuclear Techniques, Antananarivo, Madagascar \\ ${ }^{3}$ Department of Physics, Faculty of Sciences, University of Antananarivo, Antananarivo, Madagascar \\ ${ }^{4}$ Department of Physics, Faculty of Sciences, University of Fianarantsoa, Fianarantsoa, Madagascar
}

Email address:

saidsoudjay@yahoo.fr (S. Soudjay)

${ }^{*}$ Corresponding author

\section{To cite this article:}

Said Soudjay, Vololoniaina Bernardine, Ramanandraibe Marie Jeanne, Ramanantsizehena Georgette Vololona, Raboanary Roland, Randriamora Tiana Harimalala. Application of Radiation Protection of Patients in Medical Diagnostic Radiology (Case of Public Hospitals, Fianarantsoa District, Haute Matsiatra Region, Madagascar). Nuclear Science. Vol. 6, No. 1, 2021, pp. 1-4. doi: $10.11648 /$ j.ns.20210601.11

Received: January 7, 2021; Accepted: January 25, 2021; Published: March 4, 2021

\begin{abstract}
To strengthen patient radiation protection and control of medical exposure, in the Haute Matsiatra region, Fianarantsoa District, Madagascar, we have carried out a study to develop a protocol for dose assessment in diagnostic radiology in order to ensure good radiological practice. In this region, no studies have ever been done in their radiology department. The patient entrance dose $\left(D_{e}\right)$ is one of the basic dosimetric quantities for measuring the dose delivered to the patients. Therefore, to assess the patient entrance dose $\left(\mathrm{D}_{\mathrm{e}}\right)$, we have chosen two hospitals such as the Andrainjato University Hospital Center (CHUA) and the Tambavao University Hospital Center (CHUT). We have chosen four most requested radilogicals examinations (Skull, Thorax, abdomen and pelvis) and we have evaluated the entrance doses of patient and we have compared the result found to the Diagnostic Reference Levels (DRL) recommended by the IAEA for each examination. We found that, the average doses delivered to the patients during their radiographic examinations, were below the reference doses recommended by the IAEA. For this comparison carried out in these two hospitals, we can be confirmed that the values obtained can be useful for the application of regulations on the patient radiation protection and the control of medical exposure in Fianarantsoa.
\end{abstract}

Keywords: Diagnostic Radiology, Patient Entrance Dose, Radiation Protection

\section{Introduction}

Exposure to ionizing radiation can cause harmful effects not only to the subject who is exposed to it, but also to their descendants. However, the medical use of ionizing radiation must be subject to a significant regulatory framework.

The use of ionizing radiation for medical fields contributes significantly to the exposure of the population. After natural exposure, this practice presents the first source of exposure of artificial origin. A dose limitation applies to workers and the public; for patients, dose limitation is discussed case-by-case. Internationally, the setting of reference dosimetric values for diagnostic radiology examinations has been proposed. These values, called Diagnostic Reference Levels (NRL) do not constitute dose limits, but a radiological reference threshold, allowing him to locate his technique vis-a-vis the irradiation of the patient.

In Madagascar, the evaluation of the dose delivered to the patients undergoing X-ray radiological examinations was initiated in 2005 as part of the Cooperation project with the IAEA, RAF / 9/033 entitled "Strengthening radiation 
protection of patients and control of medical exposure".

At this end, we have chosen two public hospitals in Fianarantsoa such as the Andrainjato University Hospital Center (CHUA) and the Tambavao University Hospital Center (CHUT) by choosing the four most frequently used examinations (Skull, Thorax, Abdomen and Pelvis).

The objectives of this study are:

a) To study the technique used in the two hospitals center chosen to perform four types of radiological examinations common in medical radiography.

b) To collect dosimetric data and to compare them with international reference levels.

Finally, to conclude following the discussion of the results, the application of radiation protection of patients in medical diagnostic radiology in Fianarantsoa, Madagascar.

\section{Methods}

\subsection{Diagnostic Reference Levels (NRL)}

The diagnostic reference levels are the dose indicators used to assess the quality of the equipment. In the field of exposure to the ionizing radiations used for medical sectors, the regulatory limit of doses, which is one of the three principles of radiation protection, does not apply to the patient exposures. To strengthen the optimization principle, the concept of diagnostic reference level (NRL) was therefore introduced in publication 73 "Radiological Protection and Safety in medicine" of the International Commission on Radiological Protection (ICRP) [1, 5, 13, 14]. The diagnostic reference levels constitute a tool for optimization. They should not be compared with "dose limits" or "optimal doses". In practice, these levels are established for standardized examinations and typical patients.

Table 1. Diagnostic reference level for conventional radiography.

\begin{tabular}{lll}
\hline Examination & Position & $\begin{array}{l}\text { Dose at the surface by radiography } \\
(\mathbf{m G y})\end{array}$ \\
\hline Skull & PA & 5 \\
Thorax & PA & 0,4 \\
Abdomen & AP & 10 \\
Pelvis & AP & 10 \\
\hline
\end{tabular}

\subsection{Kerma (Kinetic Energy Released in Material)}

Kerma characterizes the capacity of indirectly ionizing radiation to act on matter $[13,14]$. It is the quotient of dEtr by $\mathrm{dm}$, where dEtr is the sum of the initial kinetic energies of all charged particles released by uncharged ionizing particles in a mass dm of a given material.

$$
K=\frac{d E_{t r}}{d m}
$$

The unit of kerma is Gy.

Dose in air: Da

In the reference conditions, the dose in air characterizes a radiological facility. Generally, it is measured with an ionization chamber but it can be calculated. The dose in air $\mathrm{Da}$, expressed in $\mathrm{mGy} / \mathrm{mAs}$, is given by formula 2 :

$$
D_{a}(d)=k \frac{U^{n}}{d^{2}}
$$

with $2<$ n $<3$

$\mathrm{U}$ : high voltage applied to the tube

$\mathrm{d}$ : Tube focus distance at the measurement point

$\mathrm{k}$ : Constant depending on the facility

Kerma in air has a relation with the dose in air by formula (3):

$$
K_{a i r}=D_{a}(d) . I t
$$

Kair: Kerma in air expressed in mGy

I: Current flowing through the tube

t: Irradiation time

It: Charge (mAs)

\subsection{Determination of Entrance Dose [6-14]}

The dose in air, Da, must be measured beforehand at a given reference distance dref from the source $(\mathrm{eg} 100 \mathrm{~cm})$, for the beam dimensions used in practice. The dose at the entrance surface, De, of the patient is then calculated as a function of the source-entrance surface distance (PSD), of the quality of the radiation, for the number of mAs used, for an image according to the formula 4

$$
D_{e}=D_{a} \cdot\left(\frac{d_{r e f}}{d}\right)^{2} \cdot m A s . F R D
$$

Practical unit of $\mathrm{D}_{\mathrm{e}}$ is: $\mathrm{mGy}$.

\subsection{Data Collection}

To determine the dose in air, the load is fixed but by varying the high voltage for a given distance between the source and the thermoluminescent dosimeter. This distance was set at $100 \mathrm{~cm}$. For kerma in air, we set the high voltage and we vary the charge.

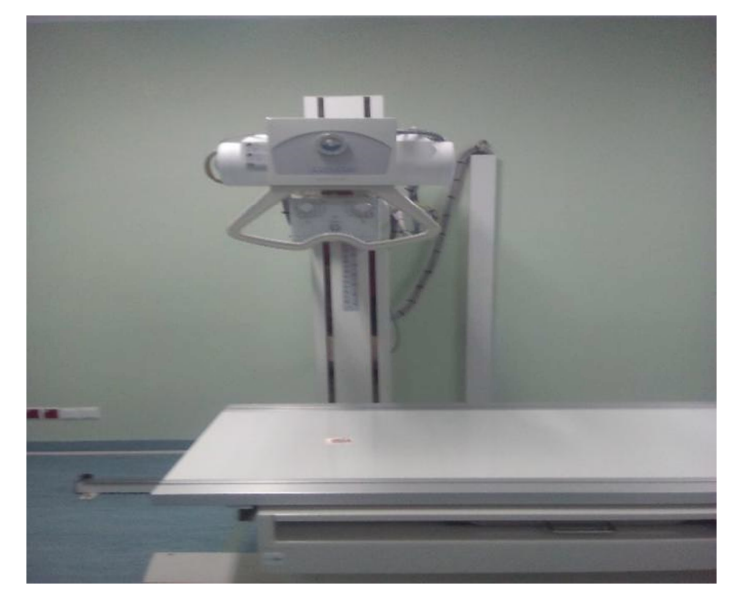

Figure 1. Experimental setup for the determination of Da and Kair. 


\section{Results}

\subsection{Dose in Air [2, 3, 5, 7, 13, 14]}

To calculate the dose received by patients during diagnostic radiology examinations, it is necessary to know the dose in air Da, the charge, the backscatter factor and the source-patient distance. For the dose in air, we set the charge of $20 \mathrm{mAs}$ and we vary the high voltage with the reference distance $100 \mathrm{~cm}$. The following table gives us the value found in the two hospitals and these values allow us to plot Figure 2.

Table 2. Dose rates of the device from both hospitals.

\begin{tabular}{lll}
\hline $\mathbf{k V}$ & Debit CHUA (mGy/mAs) & debit CHUT (mGy/mAs) \\
\hline 40 & 0,00988 & 0,00876 \\
50 & 0,01239 & 0,00994 \\
60 & 0,01831 & 0,01199 \\
70 & 0,02794 & 0,01569 \\
80 & 0,03452 & 0,02123 \\
90 & 0,04978 & 0,02956 \\
100 & 0,06523 & 0,04021 \\
\hline
\end{tabular}

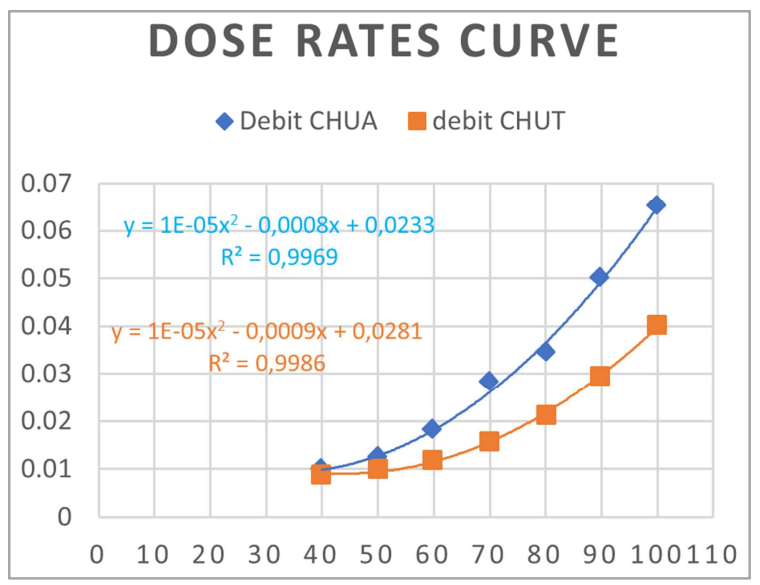

Figure 2. Dose rates curve.

\subsection{The Surface Dose for the Examinations Considered [2, $3,12-14]$}

We selected four examinations, which are considered to be the most frequent in diagnostic radiology. We considered fifteen different parameters for each type of examination. The backscatter factor is 1.35 . The distances between the X-rays tube focus and the patient's skin for CHUA are:

1. Thorax face thickness $20 \mathrm{~cm}$ : DSP (Skin-source distance) $(\mathrm{SSD})=130 \mathrm{~cm}$

2. Abdomen face thickness $20 \mathrm{~cm}$ : DSP (Skin-source distance) $(\mathrm{SSD})=130 \mathrm{~cm}$

3. Skull face thickness $15 \mathrm{~cm}$ : DSP (Skin-source distance) $(\mathrm{SSD})=85 \mathrm{~cm}$

4. Pelvis face thickness $20 \mathrm{~cm}$ : DSP (Skin-source distance) $(\mathrm{SSD})=130 \mathrm{~cm}$.

On the other hand, the distances between the X-rays tube focus and the patient's skin for CHUT are:

1. Thorax face thickness $20 \mathrm{~cm}$ : DSP (Skin-source distance) $(\mathrm{SSD})=160 \mathrm{~cm}$
2. Abdomen face thickness $20 \mathrm{~cm}$ : DSP (Skin-source distance) $(\mathrm{SSD})=90 \mathrm{~cm}$

3. Skull face thickness $15 \mathrm{~cm}$ : DSP (Skin-source distance) $(\mathrm{SSD})=85 \mathrm{~cm}$

4. Pelvis face thickness $20 \mathrm{~cm}$ : DSP (Skin-source distance) $(\mathrm{SSD})=118 \mathrm{~cm}$.

Tables 3 and 4 show the values of the doses in mGy received by the patients during their diagnostic radiology examinations in the two hospitals. We have calculated the average in order to compare of these fifteen different exams for each type of exam chosen. We will compare these average values with the NRLs and finally to know if the dose received by the patients during their radiological examination is well respected.

Table 3. Dose $(m G y)$ received by the patients at CHUA

\begin{tabular}{lllll}
\hline Examinations & Skull & Thorax & Abdomen & Pelvis \\
\hline & 0.6172 & 0.1251 & 0.2638 & 0.2638 \\
& 0.7757 & 0.1341 & 0.3316 & 0.3316 \\
& 0.8532 & 0.1453 & 0.3647 & 0.3647 \\
& 0.9308 & 0.1431 & 0.3979 & 0.3979 \\
& 0.7319 & 0.1543 & 0.3128 & 0.3128 \\
& 0.7491 & 0.1594 & 0.4171 & 0.3202 \\
& 0.8323 & 0.1905 & 0.3202 & 0.3558 \\
& 0.7664 & 0.1543 & 0.3558 & 0.3276 \\
& 0.8516 & 0.1923 & 0.3276 & 0.3641 \\
& 1.0219 & 0.1989 & 0.3640 & 0.4369 \\
& 0.7840 & 0.2321 & 0.4369 & 0.3352 \\
Average & 0.8712 & 0.2468 & 0.3352 & 0.3724 \\
& 0.9583 & 0.2384 & 0.3724 & 0.4096 \\
& 1.0454 & 0.2607 & 0.4096 & 0.4469 \\
& 1.0931 & 0.2951 & 0.4469 & 0.4571 \\
& 0.8488 & 0.1913 & 0.3638 & 0.3621 \\
\hline
\end{tabular}

Table 4. Dose ( $m G y)$ received by the patient at CHUT.

\begin{tabular}{lllll}
\hline Examinations & Skull & Thorax & Abdomen & Pelvis \\
\hline & 0.2768 & 0.1144 & 0.2469 & 0.1221 \\
& 0.2284 & 0.3502 & 0.2037 & 0.4454 \\
& 0.2575 & 0.0605 & 0.2297 & 0.7640 \\
0.3219 & 0.2579 & 0.2871 & 0.1336 \\
& 2.0284 & 0.5236 & 0.4595 & 0.1670 \\
& 0.6819 & 0.3426 & 1.8093 & 0.5346 \\
& 1.4422 & 0.2823 & 0.5433 & 1.0525 \\
& 0.5929 & 0.2907 & 0.2789 & 0.1415 \\
& 1.1421 & 0.3625 & 0.5579 & 0.5662 \\
& 2.3984 & 0.2831 & 1.1159 & 0.4677 \\
& 0.3127 & 0.2317 & 0.2864 & 1.2113 \\
& 0.3211 & 0.2371 & 0.3015 & 0.1538 \\
& 0.6762 & 0.1852 & 0.6031 & 0.1580 \\
& 0.8452 & 0.2964 & 1.2063 & 0.5071 \\
& 2.6626 & 0.2594 & 2.3750 & 0.3332 \\
& 0.9459 & 0.2718 & 0.7003 & 0.4505 \\
\hline
\end{tabular}

\section{Discussion}

From Table 2, we have presented Figure 2 illustrating the variation of the dose rates of radiological devices as a function of the voltage applied to the X-ray tube for each hospital. The curves obtained are an adjustment of the data to a polynomial function of the second degree, that is to say an equation of the form $y=a x^{2}+b x+c$. The results confirm that the dose rate in air follows the relationship mentioned in the relationship (2) 
$[13,14]$. By linear interpolation we will calculate the dose in air Da for the intermediate values which will allow us to calculate the De for each different examination.

From relation (4), we have calculated the entrance dose De with the data on the dose rates of the device. The results are given in Table 3 for CHUA and in Table 4 for CHUT.

For the CHUA, the average of the calculated dose, for each type of examination in mGy, it is compared with the NRL values (Skull: $0.8488<5$; Thorax: $0.1913<0.4$; Abdomen: $0.3638<10$; Pelvis: $0.3621<10$ ) is below the NRLs proposed by the IAEA and no examination has exceeded these NRLs. At CHUT, the average dose for each examination (Skull: $0.9459<5$; Thorax: 0.2718<0.4; Abdomen: 0.7003<10; Pelvis: $0.4505<10$ ) We found that none of the average scores for these four types of exams at the two hospitals were above the IEA suggested DRLs. On the other hand, we note that a single chest examination was exceeded, which does not mean the overall result.

However, we will be able to confirm a good practice on patient dosimetry in these two diagnostic radiology services. This proves a good application of radiation protection for patients $[1-4,12-14]$.

\section{Conclusion}

Optimization of doses delivered to the patients is regulated in Madagascar and must be controlled. In this work, we were able to estimate the doses received by the patients who underwent the four radiological examinations chosen in the two hospitals of Fianarantsoa. We have found that the average doses delivered to the patients are below the reference levels recommended by the IAEA [16]. A slight non-significant increase on the examination of the thorax at the Tambavao University Hospital Center (CHUT) is observed. The results obtained prove that radiation protection of patients is applied in these hospitals and follows the regulatory plan of the Law No. 97-041 relating to the protection against the harmful effects of ionizing radiation and radioactive waste management in Madagascar, was enacted on January 2, 1998. These results analyzed according to the influence factors on the dose, that is to say the exposure parameters and the geometric parameters, prove the feasibility and the great interest of a national campaign of dose measurements in radiology, on a larger scale, which would make it possible to establish NRLs specific to Malagasy practice, to provide for their periodic review and to define the appropriate optimization procedures. From this study, we hope to establish a digital radiological monitoring platform ensuring real-time evaluation of the doses received by the patients in the Malagasy territor.

\section{References}

[1] H. BEAUVAIS-MARCH, M. VALERO, A. BIAU, M. BOURGUIGNON; Niveaux de Référence Diagnostiques: spécificités de la démarche française en radiologie, 2003.
R. Rosa, A. L. Lassance Cunha; Body Organ Doses in Conventional Radiology in Rio de Janeiro.

[3] E. K. Ofori, W. K. Antwi, L. Arthur and H. Duah; Comparison Of Patient Radiation Dose From Chest And Lumbar Spine XRay Examinations In 10 Hospitals In Ghana, 2012.

[4] Olivera Ciraj-Bjelac, Adnan Beganovic, Dario Faj, Vesna Gershan, Sonja Ivanovic, Ivan R. Videnovic, Madan M. Rehani; Radiation protection of patients in diagnostic radiology: Status of practice in fivenEastern-European countries, based on IAEA project; Science Direct; 2011.

[5] Kate Matthews, Patrick C. Brennan; The application of diagnostic reference levels: General principles and an Irish perspective; Science Direct; 2008.

[6] European Commission. European Guidelines on quality criteria for diagnostic radiographic images, EUR 16260 EN EN, Brussels, 1996.

[7] Harpen MD. A mathematical spread sheet application for production of entrance skin exposure. Med Phys, 1996; 23 (2) 241-242.

[8] International Atomic Energy Agency. Radiation Doses in diagnostic radiology and methods for dose reduction, IAEATECDOC-796, Vienna-Austria, 1995.

[9] International Atomic Energy Agency. International Basic Safety Standards for Protection against Ionizing Radiation and for the safety of Radiation Sources, IAEA Safety Series $n^{\circ} 115$, Vienna-Austria, 1996.

[10] International Commission on Radiological Protection, Protection of the patients in diagnostic radiology. ICRP Publication 34, Pergamon Press, New York, 1982.

[11] International Commission on Radiological Protection, 1990 recommendations of ICRP, Publication 60, Pergamon Press, New York, 1991.

[12] International Commission on Radiological Protection, Radiological and Safety in Medicine, Publication 73, Pergamon Press, New York, 1996.

[13] Said Soudjay. Evaluation de la performance physique des dosimeters thermoluminescents pour la dosimétrie du patient en radiologie, Mémoire de DEA réalisé à Institut National des Sciences et Techniques Nucléaires (INSTNMADAGASCAR), Faculté des Sciences, Université d'Antananarivo, 2007.

[14] Said Soudjay, Ramanandraibe Marie Jeanne, Ramanantsizehena Georgette Vololona, Raboanary Roland, Randriantseheno Hery Fanja, Rabesiranana Naivo, Randriamora Tiana Harimalala; Efficiency of Thermoluminescents Dosimeters for Patient Dose Assessment in Medical Radiology in Antananarivo, Science Publishing Group, 2019.

[15] Eric Kwasi Ofori, Diane Scutt, Matt Ward, William Kwadwo Antwi, Edmund Kwadwo Brakohiapa, Dzefi-Tettey, Klenam, Hewlett Kwaku Vincent; Estimation Of Patient Radiation Dose From Skull X-Ray Examinations In Ghana (Running Title: Patient Dose In Skull Examinations); Insuderc Academic Publishers; 2012.

[16] IAEA; Implementation of The International Code Of Practice On Dosimetry In Diagnostic Radiology; Vienna, 2011.

[2] K. E. M. Mohamadain, A. O. Osibote, A. C. P. Azevedo, L. A. 